\title{
Generalized Thermal Flux Flow for Jeffrey Fluid with Fourier Law over an Infinite Plate
}

\author{
Muhammad Imran Asjad, ${ }^{1}$ Abdul Basit ${ }^{D},{ }^{1}$ Ali Akgül $\left(D^{2},{ }^{2}\right.$ and Taseer Muhammad ${ }^{3}$ \\ ${ }^{1}$ Department of Mathematics, University of Management and Technology Lahore, Lahore 54770, Pakistan \\ ${ }^{2}$ Siirt University, Art and Science Faculty, Department of Mathematics, 56100 Siirt, Turkey \\ ${ }^{3}$ Department of Mathematics, College of Sciences, King Khalid University, Abha 61413, Saudi Arabia \\ Correspondence should be addressed to Ali Akgül; aliakgul@siirt.edu.tr
}

Received 30 June 2021; Accepted 28 August 2021; Published 13 September 2021

Academic Editor: Fahd Jarad

Copyright (C) 2021 Muhammad Imran Asjad et al. This is an open access article distributed under the Creative Commons Attribution License, which permits unrestricted use, distribution, and reproduction in any medium, provided the original work is properly cited.

\begin{abstract}
The unsteady flow of Jeffrey fluid along with a vertical plate is studied in this paper. The equations of momentum, energy, and generalized Fourier's law of thermal flux are transformed to non-dimensional form for the proper dimensionless parameters. The Prabhakar fractional operator is applied to acquire the fractional model using the constitutive equations. To obtain the generalized results for velocity and temperature distribution, Laplace transform is performed. The influences of fractional parameters $\alpha, \beta, \gamma$, thermal Grashof number Gr, and non-dimensional Prandtl number Pr upon velocity and temperature distribution are presented graphically. The results are improved in the form of decay of energy and momentum equations, respectively. The new fractional parameter contains the Mittag-Leffler kernel with three fractional parameters which are responsible for better memory of the fluid properties rather than the exponential kernel appearing in the Caputo-Fabrizio fractional operator. The Prabhakar fractional operator has advantage over Caputo-Fabrizio in the real data fitting where needed.
\end{abstract}

\section{Introduction}

Non-Newtonian fluids have recently become more appropriate for technical and scientific applications than Newtonian fluids. Because the highly non-linear behavior of nonNewtonian fluid has a complex character, the use of nonNewtonian fluids in industries is more complicated. The study of non-Newtonian fluid has got much attention in the discipline of science and engineering due to their vast range of industrial applications, production of polymers, chemical industry, lubricant performance, food processing, and biological fluid movement. To describe the viscous behavior of these fluids, various approaches were presented. A subclass of these fluids, namely, Jeffrey fluid, has attracted a lot of attention in the last few years. Because its constitutive equation can be reduced to that of the Newtonian model as a specific case, the Jeffrey model is considered as an extension of the widely used Newtonian fluid model. The Jeffrey fluid model can represent the stress relaxation property of non-
Newtonian fluids, but the viscous fluid model is unable to describe this phenomenon. The influences of relaxation and retardation are demonstrated through the Jeffrey fluid. Hayat and Mustafa [1] examined the impact of heat transfer on free convective flow of Jeffrey fluid through porous vertical stretching sheet. Maqbool et al. [2] investigated the behavior of velocity and temperature profiles in a natural convection heat transfer of MHD Jeffrey fluid with a flat plate containing porous material using the Laplace transform. Aleem et al. [3] studied the MHD free convection transport of Jeffrey fluid between two warmed vertical plates mounted in porous medium under the influence of electric field.

Jeffrey fluid is not just a basic theoretical concept, but this is also used to solve a variety of practical difficulties, such as clay rotational motion and heart vessel pumping. Also, many scientists and researchers looked at how porosity and magnetic fields affected flow behavior in many forms of Jeffrey fluid. The Jeffrey model is a linear model that uses 
time derivatives rather than convected derivatives. In the presence of entropy generation, an unsteady two-dimensional boundary layer flow and thermal expansion of a Jeffrey fluid across a stretching sheet are addressed in [4-7]. Non-Newtonian fluid boundary layer flows across a stretching sheet, as well as thermal conductivity properties, are useful in engineering. Hot rolling, metal spinning, polymerization, sheet extrusion, and other processes are illustrations of such flows. The generalized results of peristaltic flow of the Jeffrey fluid in a cross-sectional area of a triangular duct with slip at the peristaltic boundary are obtained in [8]. There are a few further efforts for the Jeffrey fluid in [9-15].

In science and engineering, fractional calculus plays a vital role. The analysis of generalized forms of integration and differentiation is known as fractional calculus. Fractional calculus is used in a lot of different areas, including viscoelasticity, nanotechnology, electrical engineering, mechanics, signal processing, operations research, fluid mechanics, and physics [16]. Fractional-order PDE coordinate contains many physical singularities of fluid dynamics, general relativity, natural ecosystems, electrical energy, natural processes, and other interpretations. The fractional operators Caputo and Caputo-Fabrizio are defined in $[17,18]$. In fact, Michele Caputo and Mauro Fabrizio's fractional differentiation does not solve the problem of singularity and locality. This is because the kernel is integral that is non-singular, whereas Atangana and Baleanu's fractional differentiation has overcome the aforementioned shortcomings and deficiencies. The generalized MittagLeffler function is used as a kernel in this fractional differentiation, which overcomes the non-singularity and nonlocality problems. Shahid [19] obtained the generalized solution for the equations of energy and momentum along with a vertical flat plate of free convection flow with the techniques of Caputo fractional derivative. Khan et al. [20] used the techniques of Caputo fractional operator to find the exact solutions of the momentum equation of Casson fluid across an oscillating plate. CF fractional operator is used to find the effective results for an exponential function. Ali et al. [21] attained the generalized results for the incompressible flow of a Walters' B fluid across an infinite plate with the help of CF fractional operator. Asjad et al. [22] presented work on the unsteady flow of improved fluid with the techniques of fractional derivatives, namely, Caputo and Caputo-Fabrizio fractional operators. Also, the effects of different parameters on these fractional operators are justified. Murtaza et al. [23] expressed the study of Jeffrey fluid model along with the viscous dissipation, by using the Atangana-Baleanu time-fractional derivative. This idea was beneficial to find the exact solutions to mathematical problems that contain non-linear terms in the equation. The influences of diffusion-thermo, heat transfer, and magnetic field on the time-dependent MHD flow of Jeffrey nanofluid past a porous medium in a rotating frame are considered in [15]. The effects of porous media and a transversal magnetic field on a generalized Jeffrey nanofluid in a rotary vacuum system were investigated using $\mathrm{CF}$ and $\mathrm{ABC}$ time-fractional derivatives in [24]. Roohi et al. [25] inspected the unsteady free convection of an incompressible, Jeffrey nanofluid in the vicinity of a vertical oscillating plate in a porous medium. The recent studies on fractional derivatives can be seen in [26-28].

The Prabhakar integral is derived by expanding the Riemann-Liouville integral operator's kernel to include the three-parameter Mittag-Leffler function. The Prabhakar derivative is defined by substituting the Riemann-Liouville integral operator with the Prabhakar integral operator in the Riemann-Liouville derivative. Prabhakar fractional operator is used to explain certain odd habits in disordered people materials that are non-linear and non-local. The need for Prabhakar operators to specific fractional coefficients may be a useful tool for determining an appropriate statistical method that produced a strong agreement between theoretical and experimental findings. Gara and Garrappa [29] discussed the main properties of Prabhakar functions, also known as three-parametric Mittag-Leffler functions. Garra et al. [30] described how the Prabhakar fractional derivatives may be used in conventional model like the equation of temperature and magnetic dipole laser, as well as in differential equations that govern the behavior of stochastic processes.

Recently, Shah et al. [31] obtained the generalized solution for the convective flow of Maxwell fluid for the stressshear rate and heat flux density vector along with a vertical heated wall via Prabhakar fractional operator. Elnaqeeb et al. [32] used the Prabhakar fractional operator with constant wall temperature to study the carbon nanotube technique for viscous fluids and obtained results for a viscous fluid with the help of Laplace transform. Basit et al. [33] also used the same technique for second grade using the Prabhakar fractional derivative. Asjad et al. [34] used Prabhakar-like energy transport to study the convective flow of Maxwell fluid with an erect heated plate.

Khan [35] investigated the natural convection flow of Jeffrey fluid along with the vertical plate. The solutions for shear stress and velocity profile were obtained from Laplace transform. Also, it was found that the obtained results can be easily reduced to similar solutions for Newtonian fluid. Saqib et al. [36] used the Caputo-Fabrizio fractional derivative to find the exact solutions of convection flow of Jeffrey fluid. They transformed the classical model to a fractional model known as the generalized Jeffrey fluid model with the applications of the Caputo-Fabrizio derivative. The Laplace transform was used to get closed-form solutions.

Therefore, in view of the aforementioned recent literature, no study has been discussed so far for Jeffrey fluid with Prabhakar fractional derivative. So, in order to fill this research gap, we intended to obtain the natural convection flow of Prabhakar fractional derivative through generalized thermal flux of Jeffrey fluid along with an infinite vertical plate with a constant temperature. On the basis of generalized fractional constitutive equations, we present a mathematical model for the stress relaxation property. The analytical solutions are obtained with the Laplace transform method and compared with recent published work for the validation of the present results. 


\section{Mathematical Formulation}

Assume an unsteady convective flow of Jeffrey fluid along with an infinite vertical plate situated at $y=0$ in the $x$-direction while $y$-direction is normal to the plate. For $t=0$, both the infinite plate and fluid are stationary at ambient temperature $T_{\infty}$. After $t>0$, the temperature of the plate rose to $T_{w}$ which can be seen in Figure 1 .

The constitutive equations for a Jeffrey fluid are as follows [35]:

$$
\begin{aligned}
T & =-p I+S, \\
S & =\frac{\mu}{1+\lambda}\left(A+\lambda_{1} \frac{\mathrm{d} A}{\mathrm{~d} t}\right), \\
\frac{\mathrm{d}}{\mathrm{d} t} & =\frac{\partial}{\partial t}+\nabla \cdot V,
\end{aligned}
$$

where $-p I$ is indeterminate part of the stress, $S$ is extra stress tensor, and $\lambda$ and $\lambda_{1}$ are the material parameters of Jeffrey fluid.

The Rivlin-Ericksen tensor $A$ is defined as

$$
\begin{aligned}
A & =L+L^{T}, \\
L & =\nabla V .
\end{aligned}
$$

For unidirectional and one-dimensional flow velocity, temperature and stress fields are given as

$$
\begin{aligned}
V & =\widetilde{u}(\tilde{y}, \widetilde{t}) i, \\
S & =S(\widetilde{y}, \widetilde{t}), \\
T & =\widetilde{T}(\widetilde{y}, \widetilde{t}),
\end{aligned}
$$

where $i$ represents the unit vector along $x$-axis and the stress field satisfies the condition $S(\tilde{y}, 0)=0$. Hence, we obtain $S_{x x}=S_{y y}=S_{z z}=S_{x z}=S_{z x}=S_{y z}=S_{z y}=0$ and

$$
S_{x y}=\frac{\mu}{1+\lambda}\left(1+\lambda_{1} \frac{\partial}{\partial \widetilde{t}}\right) \frac{\partial \widetilde{u}}{\partial \widetilde{y}}
$$

where $S_{x y}$ is the non-trivial tangential stress.

According to these observations and using equation (4), the continuity equation satisfies identically. Using equations (1)-(5) and Boussinesq approximation, the equations of momentum, energy, and classical Fourier's law of thermal flux for time dependent and free convective stream of Jeffrey fluid over a vertically situated plate are obtained by the following [36].

Momentum equation:

$$
\rho \frac{\partial \widetilde{u}}{\partial \widetilde{t}}=\frac{\mu}{1+\lambda}\left(1+\lambda_{1} \frac{\partial}{\partial \widetilde{t}}\right) \frac{\partial^{2} \widetilde{u}}{\partial \widetilde{y}^{2}}+g \rho \beta_{T}\left[\widetilde{T}-T_{\infty}\right] .
$$

Constitutive energy equation:

$$
\rho C_{p} \frac{\partial \widetilde{T}}{\partial \widetilde{t}}=-\frac{\partial \widetilde{q}}{\partial \widetilde{y}} .
$$

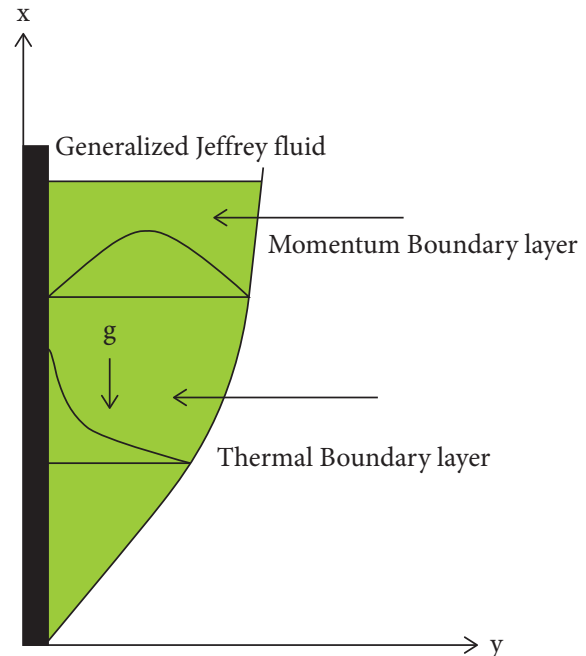

Figure 1: Model figure of the problem.

For this motive, we utilize the Prabhakar fractional operator based on generalized Fourier's law [34].

$$
\widetilde{q}=-k^{C} D_{\alpha, \beta, a}^{\gamma} \widetilde{T}_{\tilde{y}},
$$

where $\widetilde{u}$ denotes the velocity field, $\widetilde{T}$ is the fluid temperature, $\tilde{q}$ is the thermal flux, and all the physical variables are functions of $\tilde{y}$ and $\tilde{t}$.

The constraints for velocity and temperature are given as follows:

$$
\begin{aligned}
& \text { for } \widetilde{t}=0, \widetilde{u}=0, \widetilde{T}=T_{\infty}, \widetilde{y}>0, \\
& \text { for } \widetilde{y}=0, \widetilde{u}=0, \widetilde{T}=T_{w}, \widetilde{t}>0,
\end{aligned}
$$$$
\text { for } \tilde{y} \longrightarrow \infty, \tilde{u}=0, \widetilde{T} \longrightarrow T_{\infty}, \widetilde{t}>0 \text {. }
$$

Proposing dimensionless parameters

$$
\begin{aligned}
& y=\frac{u_{o}}{v} \widetilde{y}, \\
& t=\frac{u_{o}^{2}}{v} \widetilde{t}, \\
& u=\frac{\widetilde{u}}{u_{o}}, \\
& \theta=\frac{\widetilde{T}-T_{\infty}}{T_{w}-T_{\infty}}, \\
& q=\frac{\widetilde{q}}{q_{0}}, \\
& q_{0}=\frac{k\left(T_{w}-T_{\infty}\right) u_{o}}{\nu},
\end{aligned}
$$


into the equations (6)-(11), we can get

$$
\begin{aligned}
\frac{\partial u}{\partial t} & =\frac{1}{1+\lambda}\left(1+\lambda_{2} \frac{\partial}{\partial t}\right) \frac{\partial^{2} u}{\partial y^{2}}+\mathrm{Gr} \theta \\
\frac{\partial \theta}{\partial t} & =-\frac{1}{\operatorname{Pr}} \frac{\partial q}{\partial y}, \\
q & =-{ }^{C} D_{\alpha, \beta, a}^{\gamma} \theta_{y},
\end{aligned}
$$

where the dimensionless constraints are given by

$$
\begin{aligned}
& \text { for } t=0, u=\theta=0, \\
& \text { for } y=0, u=0, \theta=1, \\
& \text { for } y \longrightarrow \infty, u=\theta \longrightarrow 0,
\end{aligned}
$$

where $\operatorname{Pr}=\left(\mu C_{p} / k\right)$ is dimensionless Prandtl number, $\mathrm{Gr}=$ $\left(\left(T_{w}-T_{\infty}\right) g v \beta / u_{0}^{3}\right)$ is thermal Grashof number, and $\lambda_{2}=$ $\left(u_{o}^{2} / \nu\right) \lambda_{1}$ is the non-dimensional Jeffrey fluid parameter.

As a result, the preliminaries of fractional calculus are taken into account for mathematical modelling, needed for this work.

\section{Preliminaries of Fractional Calculus}

Definition 1. (the Prabhakar kernel). Function [34]

For $t \in R$,

$$
e_{\alpha, \beta}^{\gamma}(a ; t)=t^{\beta-1} E_{\alpha, \beta}^{\gamma}\left(a t^{\alpha}\right), \quad \alpha, \beta, \gamma, a \in C, \operatorname{Re}(\alpha)>0,
$$

is known as Prabhakar kernel.

Definition 2. (the Prabhakar integral). Function [34]

For $t \in(0, b)$ and a function $h \in L^{1}(0, b)$,

$$
E_{\alpha, \beta, a}^{\gamma} h(t)=h(t) * e_{\alpha, \beta}^{\gamma}(a ; t)=\int_{0}^{t} h(\tau)(t-\tau)^{\beta-1} E_{\alpha, \beta}^{\gamma}\left(a(t-\tau)^{\alpha}\right) \mathrm{d} \tau
$$

is called Prabhakar integral. Laplace transform of equation (20) is given by

$$
\begin{aligned}
L\left\{E_{\alpha, \beta, a}^{\gamma} h(t)\right\}(s) & =L\{h(t)\} L\left\{e_{\alpha, \beta}^{\gamma}(a ; t)\right\} \\
& =L\{h(t)\} \frac{s^{\alpha \gamma-\beta}}{\left(s^{\alpha}-a\right)^{\gamma}} .
\end{aligned}
$$

Definition 3. (the Prabhakar derivative). Let $g \in L^{1}(0, b)$ and $\tau \in(0, b)$, then for $m=[\nu]$. The Prabhakar derivative is defined by [34]

$$
\left(D_{\alpha, \nu, \beta, a^{+}}^{\gamma} g\right)(\tau)=\left(\frac{\mathrm{d}^{m}}{\mathrm{~d} t^{m}}\left(E_{\alpha, n-\gamma, \beta, a^{+}}^{-\gamma} g\right)\right)(\tau),
$$

where $\alpha, \beta, \gamma, \nu$ are the elements of complex number $C$.
Definition 4. (the regularized Prabhakar derivative). Let $0<\beta \leq n$, where $n$ belongs to $Z$ and $h \in A C^{n}(0, b)$. The regularized Prabhakar derivative is defined as $[29,34]$

$$
\begin{aligned}
{ }^{c} D_{\alpha, \beta, a}^{\gamma} h(t) & =h^{(n)}(t) E_{\alpha, n-\beta, \alpha}^{-\gamma}=h^{(n)}(t) * e_{\alpha, n-\beta}^{-\gamma}(a ; t) \\
& =\int_{0}^{t} h^{(n)}(\tau)(t-\tau)^{n-\beta-1} E_{\alpha, n-\beta}^{-\gamma}\left(a(t-\tau)^{\alpha}\right) \mathrm{d} \tau,
\end{aligned}
$$

where $h^{(n)}$ represents the nth differential of $h(t)$ and $A C^{n}(0, b)$ represents the set of functions $h(t)$ with real values. Laplace transform of the equation (23) is given by

$$
\begin{aligned}
L\left\{{ }^{c} D_{\alpha, \beta, a}^{\gamma} h(t)\right\} & =L\left\{e_{\alpha, n-\beta}^{-\gamma} * h^{(n)}(t)\right\} \\
& =L\left\{e_{\alpha, n-\beta}^{-\gamma}(a ; t)\right\} L\left\{h^{(n)}(t)\right\} \\
& =s^{\beta-n}\left(1-a s^{-\alpha}\right)^{\gamma} L\left\{h^{(n)}(t)\right\} .
\end{aligned}
$$

\section{Solution of the Problem}

4.1. Solution of the Temperature. By Laplace transform, equations (14) and (15), with the constraints of equation (16), can be transformed to achieve the solution of fluid temperature.

$$
\begin{aligned}
s \bar{\theta}(y, s)+\frac{1}{\operatorname{Pr}} \frac{\partial \bar{q}(y, s)}{\partial y} & =0, \\
\bar{q}(y, s)+s^{\beta}\left(1-a s^{-\alpha}\right)^{\gamma} \frac{\partial \bar{\theta}(y, s)}{\partial y} & =0, \\
\bar{\theta}(0, s)-\frac{1}{s} & =0, \\
\bar{\theta}(\infty, s) & \longrightarrow 0 .
\end{aligned}
$$

By introducing equation (26) into equation (25), we have

$$
\frac{\partial^{2} \bar{\theta}(y, s)}{\partial y^{2}}-\frac{\operatorname{Prs}}{s^{\beta}\left(1-a s^{-\alpha}\right)^{\gamma}} \bar{\theta}(y, s)=0
$$

and solution of equation (28) with respect to the constraints given in equation (27). Then, we get

$$
\bar{\theta}(y, s)=\frac{1}{s} e^{-y \sqrt{\left(\operatorname{Prs}^{1-\beta /\left(1-a s^{-\alpha}\right)^{\gamma}}\right)}}
$$

or

$$
\bar{\theta}(y, s)=\frac{1}{s}+\frac{1}{s} \sum_{u=1}^{\infty} \frac{\left(-y \sqrt{\left.\left(\operatorname{Pr} s^{1-\beta /\left(1-a s^{-\alpha}\right.}\right)^{\gamma}\right)}\right)^{u}}{u !} .
$$

In order to obtain the inverse Laplace transform of equation (30), it can be transformed into appropriate form by using the formula $(1-x)^{-u}=\sum_{v=0}^{\infty} x^{v}(\sqrt{(u+v)} / v !(\sqrt{u}))$.

$$
\bar{\theta}(y, s)=\frac{1}{s}+\sum_{u=1}^{\infty} \sum_{v=0}^{\infty} \frac{(-y)^{u}(\operatorname{Pr})^{(u / 2)} a^{v}(\sqrt{(u / 2)+v})}{u ! v ! s^{1+(\beta u / 2)-(u / 2)+\alpha v} \sqrt{(u / 2)}} .
$$


The inverse Laplace transform of equation (31) can be found by $L^{-1}\left\{\Gamma(k) / s^{k}\right\}=t^{k-1}, k \geq 0$.

$$
\theta(y, t)=1+\sum_{u=1}^{\infty} \sum_{v=0}^{\infty} \frac{(-y)^{u}(\operatorname{Pr})^{(u / 2)} a^{v} t^{(\beta u / 2)-(u / 2)+\alpha v} \sqrt{((u / 2)+v)}}{u ! v ! \sqrt{(1+(\beta u / 2)-(u / 2)+\alpha v)} \sqrt{(u / 2)}} .
$$

Nusselt Number. In order to calculate the rate of heat transfer from the plate to the fluid in Nusselt number form, the following expression can be used.

$$
\mathrm{Nu}=-\left.\frac{\partial \theta(y, t)}{\partial y}\right|_{y=0} .
$$

4.1.1. Classical Solution of Temperature $(\beta=\gamma=0)$. In the present work, we consider $\beta \in[0,1)$ and the parameter $n=0$. Then, equation (24) can be reduced to

$$
L\left\{e_{\alpha, \beta}^{-\gamma}(a ; t)\right\}=L\left\{t^{\beta-1} E_{\alpha, \beta}^{-\gamma}\left(a t^{\alpha}\right)\right\}=s^{\beta}\left(1-a s^{-\alpha}\right)^{\gamma} .
$$

For $\beta=\gamma=0$, equation (34) becomes

$$
L\left\{e_{\alpha, 0}^{0}(a ; t)\right\}=1 .
$$

In this special case, equation (15) becomes classical Fourier's law, and solution of temperature in equation (29) is

$$
\bar{\theta}(y, s)=\frac{1}{s} \exp (-y \sqrt{\operatorname{Pr} s}) .
$$

The inverse Laplace transform of equation (36) can be found by

$$
\theta(y, t)=\operatorname{erfc}\left(\frac{y}{2} \sqrt{\frac{\operatorname{Pr}}{t}}\right) .
$$

Nusselt Number. In order to compute heat transfer rate from plate to the fluid in the form of Nusselt number, the following expression can be used:

$$
\mathrm{Nu}=\left.\frac{-\partial \theta(y, t)}{\partial y}\right|_{y=0} .
$$

4.2. Solution of Velocity Field. Applying Laplace transform to equations (13), (17), and (18), with the constraints of equation (16), it can be transformed to achieve the solution of fluid velocity.

$$
\begin{gathered}
s \bar{u}(y, s)-\frac{1}{1+\lambda_{1}}\left(1+\lambda_{2} s\right) \frac{\partial^{2} \bar{u}(y, s)}{\partial y^{2}}-\operatorname{Gr} \bar{\theta}(y, s)=0, \\
\bar{u}(0, s)=\bar{u}(\infty, s)=0 .
\end{gathered}
$$

Solution of equation (39) under the constraints is given in equation (40). Thus, we have

$$
\frac{\partial^{2} u(y, s)}{\partial y^{2}}-s \frac{\left(1+\lambda_{1}\right)}{1+\lambda s} \bar{u}(y, s)=-\mathrm{Gr} \frac{\left(1+\lambda_{1}\right)}{1+\lambda s} \bar{\theta}(y, s) .
$$

Now, substituting equation (29) into equation (41), we get the result for velocity fluid.

$$
\bar{u}(y, s)=\operatorname{Gr} \frac{\left(1+\lambda_{1}\right)}{(1+\lambda s)} \frac{1}{q(s)}\left(\frac{e^{-y \sqrt{\left(1+\lambda_{1}\right) /(1+\lambda s)}}}{s}-\frac{e^{-y \sqrt{\operatorname{Prs}^{1-\beta /} /\left(1-a s^{-\alpha}\right)^{\gamma}}}}{s}\right),
$$

where

$$
q(s)=\left(\frac{\operatorname{Prs}^{1-\beta}}{\left(1-a s^{-\alpha}\right)^{\gamma}}-\frac{s\left(1+\lambda_{1}\right)}{(1+\lambda s)}\right) .
$$

The inversion Laplace transform to the equation (42) can be obtained analytically by Stehfest's and Tzou's algorithms $[37,38]$.

\section{Physical Discussion}

A modern definition of fractional derivative, namely, Prabhakar fractional operator, is defined in the recent study. The solution for temperature and velocity profile is obtained using the Laplace transform. The graphical behavior for fluid temperature can be visualized for fractional parameters $\alpha, \beta, \gamma$ and Prandtl number Pr. The influence of fractional parameters $\alpha, \beta, \gamma$ and thermal Grashof number Gr is analyzed for velocity distribution. The comparison between the fractional derivatives Caputo-Fabrizio and Prabhakar fractional operator is also justified. In Figure 2, it can be viewed that for time $t=0.2$, the fluid temperature increases by adding up the values of $\alpha, \beta, \gamma$. The reason for this phenomenon is that greater values of fractional parameters increase the buoyancy forces, and consequently, the thickness of the thermal boundary layer increases, which causes the temperature profile to rise. The influence of the nondimensional Prandtl number $\operatorname{Pr}$ on fluid temperature is shown in Figure 3. It can be seen that temperature distribution is decreased by increasing the non-dimensional Prandtl number Pr and fixing other parameters constant. The ratio of momentum diffusivity to heat diffusivity is known as the Prandtl number. As a result, fluids with lower values of Prandtl number have higher thermal conductivity, which makes the heat diffuse away from the heated surface more rapidly and faster compared to higher values of Prandtl number. Therefore, increasing the thickness of the boundary layer causes a reduction in the temperature distribution. Figure 4 shows the comparison between the fractional derivatives Caputo-Fabrizio and Prabhakar fractional operator for temperature distribution. The temperature profile of the Prabhakar fractional operator is found to be lower than the temperature distribution of Caputo-Fabrizio fractional operator.

Figure 5 visualizes the influence of velocity distribution on fractional parameters $\alpha, \beta, \gamma$. For $t=0.8$ and when the values of fractional parameters are increased while the other 


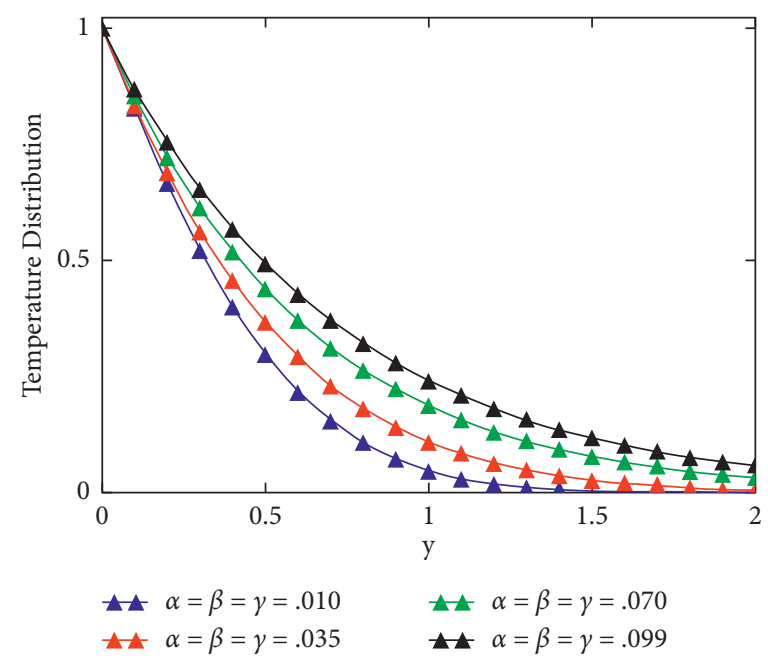

Figure 2: Temperature distribution against $y$ due to $\alpha, \beta$, and $\gamma$ when $t=0.2, \operatorname{Pr}=6.2$, and $a=0.05$.

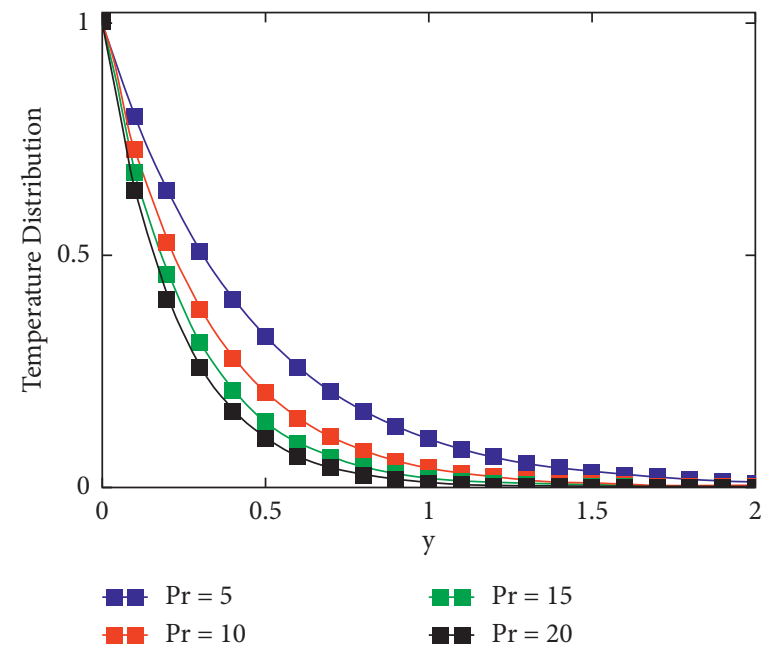

Figure 3: Temperature distribution against $y$ due to $\operatorname{Pr}$ when $t=0.2, \alpha=0.9, \beta=0.9$, and $\gamma=0.9$.

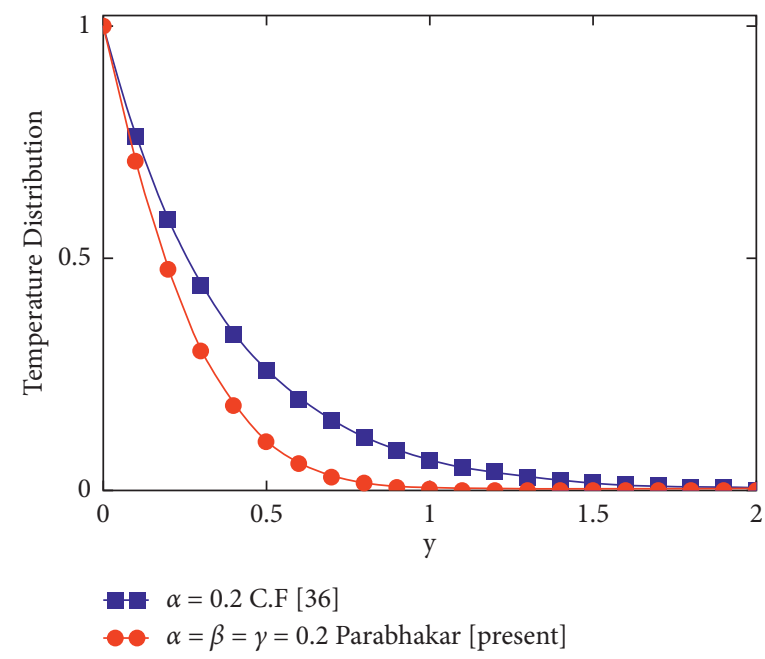

Figure 4: Temperature comparison between Caputo-Fabrizio and Prabhakar derivatives when $t=0.2, \operatorname{Pr}=6.2$, and $a=0.05$. 


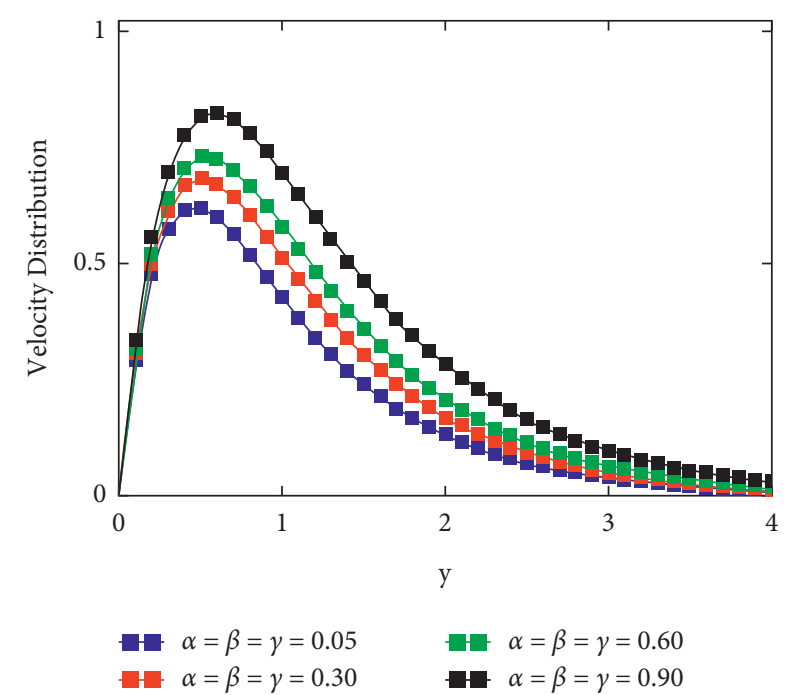

Figure 5: Velocity distribution against $y$ due to $\alpha, \beta$, and $\gamma$ when $t=0.8, \operatorname{Pr}=6.2, \mathrm{Gr}=15$, and $\lambda=0.5$.

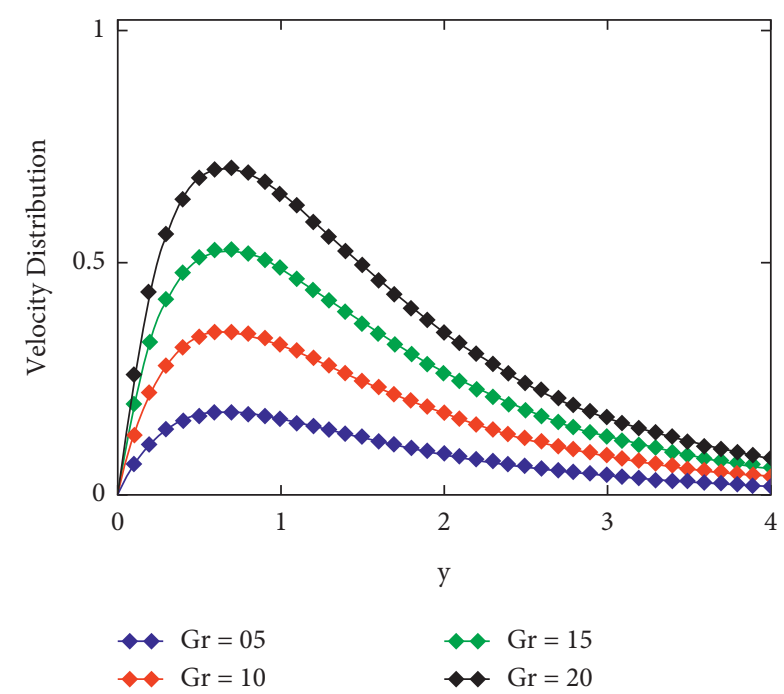

FIgURE 6: Velocity distribution against $y$ due to Gr when $t=0.8$, $\operatorname{Pr}=6.2, \alpha=0.9, \beta=0.9, \gamma=0.9$, and $\lambda=0.5$.

parameters remain constant, fluid velocity can be increased. The thickness of the boundary layer increases with time, and velocity is maximum in the region of the plate. Figure 6 indicates how the Grashof number Gr influences velocity distribution. The fluid velocity can be increased by increasing the values of Gr. Physically, the Grashof number is expressed as the ratio of buoyancy forces to viscous forces. Large values of $\mathrm{Gr}$ increase the buoyancy forces, which gives rise to an increase in the induced flow. As a result, fluid velocity can be increased. In Figure 7, the comparison between the fractional derivatives Caputo-Fabrizio and Prabhakar fractional operator on velocity distribution is justified. For larger time, the velocity profile of the fractional operator Caputo-Fabrizio is found to be higher as compared

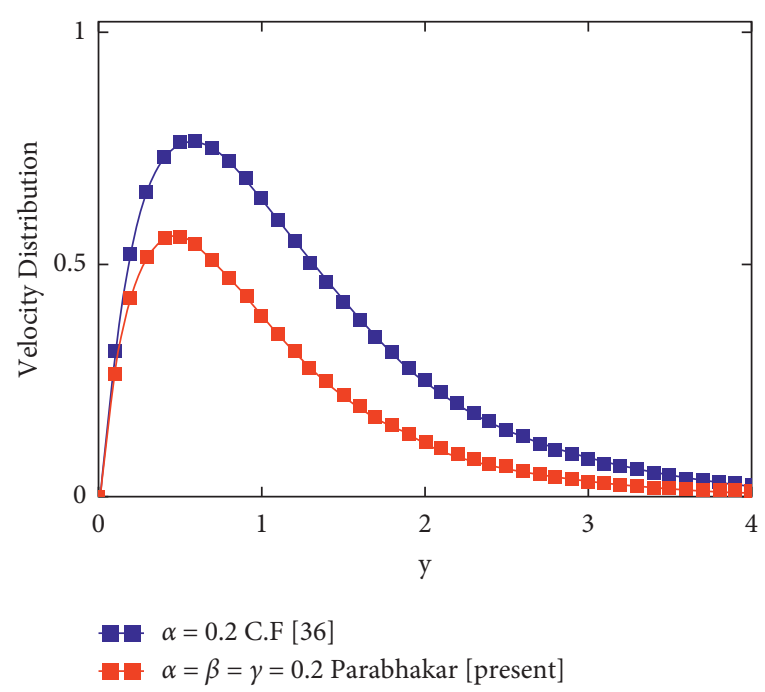

Figure 7: Velocity comparison between Caputo-Fabrizio and Prabhakar derivatives when $t=0.8, \operatorname{Pr}=6.2, \mathrm{Gr}=15$, and $\lambda=0.5$.

TABLE 1: Nomenclature.

\begin{tabular}{lcc}
\hline Name & Symbol & Unit \\
\hline Gravitational acceleration & $g$ & $\mathrm{~ms}^{-2}$ \\
Density of fluid & $\rho$ & $\mathrm{kgm}^{-3}$ \\
Viscosity of fluid & $\mu$ & $\mathrm{kgm}^{-1} \mathrm{~s}^{-1}$ \\
Heat capacity of fluid & $C_{p}$ & $j K^{-1} \mathrm{~kg}^{-1}$ \\
Thermal expansion & $\beta_{T}$ & $K^{-1}$ \\
Thermal conductivity & $k$ & $\mathrm{Wm}^{-2} \mathrm{k}^{-1}$ \\
Kinematic viscosity & $\nu$ & $\mathrm{m}^{2} \mathrm{~s}^{-1}$ \\
Temperature at infinity & $T_{\infty}$ & $K$ \\
Temperature at plate & $T_{w}$ & $K$ \\
Time & $t$ & $s$ \\
Gauss complementary error function & $\operatorname{Erfc}(\cdot)$ & \\
\hline
\end{tabular}

to the velocity obtained by Prabhakar fractional operator in the sense of memory. It is found that the new fractional parameter contains the Mittag-Leffler kernel with three fractional parameters which are responsible for better memory of the fluid properties rather than the exponential kernel appearing in Caputo-Fabrizio fractional operator.

\section{Conclusion}

In this article, the unsteady and convected stream of a Jeffrey fluid along with a vertically situated plate is solved analytically with Laplace transform. The influences of fractional parameters $\alpha, \beta, \gamma$, thermal Grashof number Gr, and Prandtl number Pr upon temperature and velocity profile are presented graphically. To find the inverse Laplace transform for velocity profile, Tzou's and Stehfest's algorithms are utilized. The major findings of this article are given as follows (Table 1):

(i) For the larger values of Pr, temperature can be decreased, whereas temperature rises as fractional parameters $\alpha, \beta, \gamma$ increase. 
(ii) Velocity can be elevated to enhance the values of thermal Grashof number $\mathrm{Gr}$ and fractional parameters $\alpha, \beta, \gamma$.

(iii) The thickness of thermal and momentum boundary layers may be controlled by using the fractional parameters with respect to different time values.

(iv) The new fractional parameter contains the MittagLeffler kernel with three fractional parameters which are responsible for better memory of the fluid properties rather than the exponential kernel appearing in Caputo-Fabrizio fractional operator.

\section{Data Availability}

The data that support the findings of this study are included within the article.

\section{Conflicts of Interest}

The authors declare that they have no conflicts of interest.

\section{Acknowledgments}

The authors extend their appreciation to the Deanship of Scientific Research at King Khalid University, Abha, Saudi Arabia, for funding this work through Research Groups Program under grant no. R.G.P-1/178/42.

\section{References}

[1] T. Hayat and M. Mustafa, "Influence of thermal radiation on the unsteady mixed convection flow of a Jeffrey fluid over a stretching sheet," Zeitschrift für Naturforschung A, vol. 65, no. 8-9, pp. 711-719, 2010.

[2] K. Maqbool, A. B. Mann, and M. H. Tiwana, "Unsteady MHD convective flow of a Jeffery fluid embedded in a porous medium with ramped wall velocity and temperature," Alexandria engineering journal, vol. 57, no. 2, pp. 1071-1078, 2018.

[3] M. Aleem, M. I. Asjad, A. Ahmadian, M. Salimi, and M. Ferrara, "Heat transfer analysis of channel flow of MHD Jeffrey fluid subject to generalized boundary conditions," The European physical journal plus, vol. 135, no. 1, pp. 1-15, 2020.

[4] M. Ali and F. Al-Yousef, "Laminar mixed convection boundary layers induced by a linearly stretching permeable surface," International Journal of Heat and Mass Transfer, vol. 45, no. 21, pp. 4241-4250, 2002.

[5] S. Mukhopadhyay, "Effect of thermal radiation on unsteady mixed convection flow and heat transfer over a porous stretching surface in porous medium," International Journal of Heat and Mass Transfer, vol. 52, no. 13-14, pp. 3261-3265, 2009.

[6] T. Hayat and M. Qasim, "Radiation and magnetic field effects on the unsteady mixed convection flow of a second grade fluid over a vertical stretching sheet," International Journal for Numerical Methods in Fluids, vol. 66, no. 7, pp. 820-832, 2011.

[7] K. Ahmad and A. Ishak, "MHD flow and heat transfer of a Jeffrey fluid over a stretching sheet with viscous dissipation," Malaysian journal of mathematical sciences, vol. 10, pp. 311323, 2016.

[8] A. Riaz, S. Nadeem, R. Ellahi, and A. Zeeshan, "Exact solution for peristaltic flow of Jeffrey fluid model in a three dimensional rectangular duct having slip at the walls," Applied Bionics and Biomechanics, vol. 11, no. 1-2, pp. 81-90, 2014.

[9] A. M. Siddiqui, A. A. Farooq, T. Haroon, and B. S. Babcock, "A variant of the classical von Karman flow for a Jeffrey fluid," Applied Mathematical Sciences, vol. 7, no. 20, pp. 983-991, 2013.

[10] S. Nadeem, A. Riaz, and R. Ellahi, "Peristaltic flow of a Jeffrey fluid in a rectangular duct having compliant walls," Chemical Industry and Chemical Engineering Quarterly, vol. 19, no. 3, pp. 399-409, 2013.

[11] A. Zeeshan and A. Majeed, "Heat transfer analysis of Jeffery fluid flow over a stretching sheet with suction/injection and magnetic dipole effect," Alexandria engineering journal, vol. 55, no. 3, pp. 2171-2181, 2016.

[12] N. A. Mohd Zin, I. Khan, and S. Shafie, "Influence of thermal radiation on unsteady MHD free convection flow of Jeffrey fluid over a vertical plate with ramped wall temperature," Mathematical Problems in Engineering, vol. 2016, no. 4, pp. 1-12, 2016.

[13] M. Kothandapani and J. Prakash, "Convective boundary conditions effect on peristaltic flow of a MHD Jeffery nanofluid," Applied Nanoscience, vol. 6, no. 3, pp. 323-335, 2016.

[14] A. Khan, G. Zaman, A. Din, and S. Muhammad, "Analytic solution of fractional Jeffrey fluid induced by abrupt motion of the plate," Matrix science mathematics, vol. 2, no. 1, pp. 1-3, 2018.

[15] F. Ali, S. Murtaza, I. Khan, N. A. Sheikh, and K. S. Nisar, "Atangana-Baleanu fractional model for the flow of Jeffrey nanofluid with diffusion-thermo effects: applications in engine oil," Advances in Difference Equations, vol. 2019, no. 1, pp. 1-21, 2019.

[16] J. A. T. M. J. Sabatier, O. P. Agrawal, and J. T. Machado, Advances in Fractional Calculus, Springer, Berlin, Germany, 2007.

[17] M. Caputo and M. Fabrizio, "A new definition of fractional derivative without singular kernel," Progress in fractional differentiation and applications, vol. 1, no. 2, pp. 73-85, 2015.

[18] M. Caputo and M. Fabrizio, "Applications of new time and spatial fractional derivatives with exponential kernels," Progress in fractional differentiation and applications, vol. 2, no. 1, pp. 1-11, 2016.

[19] N. Shahid, "A study of heat and mass transfer in a fractional MHD flow over an infinite oscillating plate," SpringerPlus, vol. 4, no. 1, pp. 640-721, 2015.

[20] I. Khan, N. Ali Shah, and D. Vieru, "Unsteady flow of generalized Casson fluid with fractional derivative due to an infinite plate," The European physical journal plus, vol. 131, no. 6, Article ID 181, 2016.

[21] F. Ali, M. Saqib, I. Khan, and N. A. Sheikh, "Application of Caputo-Fabrizio derivatives to MHD free convection flow of generalized Walters'-B fluid model," The European physical journal plus, vol. 131, no. 10, pp. 1-10, 2016.

[22] M. I. Asjad, N. A. Shah, M. Aleem, and I. Khan, "Heat transfer analysis of fractional second-grade fluid subject to Newtonian heating with Caputo and Caputo-Fabrizio fractional derivatives: a comparison," The European physical journal plus, vol. 132, no. 8, pp. 1-19, 2017.

[23] S. Murtaza, F. Ali, N. A. Aamina, N. Ahmad Sheikh, I. Khan, and K. Sooppy Nisar, "Exact analysis of non-linear fractionalized Jeffrey fluid. A novel approach of atangana-baleanu fractional model," Computers, Materials \& Continua, vol. 65, no. 3, pp. 2033-2047, 2020. 
[24] F. Ali, S. Murtaza, N. A. Sheikh, and I. Khan, "Heat transfer analysis of generalized Jeffery nanofluid in a rotating frame: Atangana-Balaenu and Caputo-Fabrizio fractional models," Chaos, Solitons \& Fractals, vol. 129, pp. 1-15, 2019.

[25] R. Roohi, M. H. Heydari, and H. G. Sun, "Numerical study of unsteady natural convection of variable-order fractional Jeffrey nanofluid over an oscillating plate in a porous medium involved with magnetic, chemical and heat absorption effects using Chebyshev cardinal functions," The European Physical Journal Plus, vol. 134, no. 10, pp. 1-17, 2019.

[26] M. I. Asjad, M. D. Ikram, and A. Akgül, "Analysis of MHD viscous fluid flow through porous medium with novel power law fractional differential operator," Physica Scripta, vol. 95, no. 11, Article ID 115209, 2020.

[27] M. B. Riaz, J. Awrejcewicz, and D. Baleanu, "Exact solutions for thermomagetized unsteady non-singularized Jeffrey fluid: effects of ramped velocity, concentration with Newtonian heating," Results in physics, vol. 26, Article ID 104367, 2021.

[28] F. Wang, M. I. Asjad, M. Zahid, A. Iqbal, H. Ahmad, and M. D. Alsulami, "Unsteady thermal transport flow of Casson nanofluids with generalized Mittag-Leffler kernel of Prabhakar's type," Journal of materials research and technology, vol. 14, pp. 1292-1300, 2021.

[29] R. Garra and R. Garrappa, "The Prabhakar or three parameter Mittag-Leffler function: theory and application," Communications in Nonlinear Science and Numerical Simulation, vol. 56, pp. 314-329, 2018.

[30] R. Garra, R. Gorenflo, F. Polito, and Ž. Tomovski, "HilferPrabhakar derivatives and some applications," Applied Mathematics and Computation, vol. 242, pp. 576-589, 2014.

[31] N. A. Shah, C. Fetecau, and D. Vieru, "Natural convection flows of Prabhakar-like fractional Maxwell fluids with generalized thermal transport," Journal of Thermal Analysis and Calorimetry, pp. 1-14, 2020.

[32] T. Elnaqeeb, N. A. Shah, and I. A. Mirza, "Natural convection flows of carbon nanotubes nanofluids with Prabhakar-like thermal transport," Mathematical Methods in the Applied Sciences, 2020.

[33] A. Basit, M. I. Asjad, and A. Akgül, "Convective flow of a fractional second grade fluid containing different nanoparticles with Prabhakar fractional derivative subject to nonuniform velocity at the boundary," Mathematical Methods in the Applied Sciences, 2021.

[34] M. I. Asjad, A. Basit, A. Iqbal, and N. A. Shah, "Advances in transport phenomena with nanoparticles and generalized thermal process for vertical plate," Physica Scripta, vol. 96, no. 11, Article ID 114001, 2021.

[35] I. Khan, "A note on exact solutions for the unsteady free convection flow of a Jeffrey fluid," Zeitschrift für Naturforschung A, vol. 70, no. 6, pp. 397-401, 2015.

[36] M. Saqib, F. Ali, I. Khan, N. A. Sheikh, S. A. A. Jan, and fnm Samiulhaq, "Exact solutions for free convection flow of generalized Jeffrey fluid: a Caputo-Fabrizio fractional model," Alexandria engineering journal, vol. 57, no. 3, pp. 1849-1858, 2018.

[37] H. Stehfest, "Algorithm 368: numerical inversion of Laplace transforms [D5]," Communications of the ACM, vol. 13, no. 1, pp. 47-49, 1970.

[38] D. Y. Tzou, Macro-to Microscale Heat Transfer: The Lagging Behavior, John Wiley \& Sons, Hoboken, NJ, USA, 2014. 